\section{Somatic growth in girls receiving low dose prophylactic co-trimoxazole}

Recurrence of urinary tract infection is common in childhood and indicates some impairment of the defences of the bladder. Provided that there is no outflow obstruction, reinfections of the urinary tract may be effectively prevented by using an appropriate prophylactic antibacterial agent until the underlying cause resolves or is corrected.' Co-trimoxazole has been used effectively in low dosage for this purpose but, in view of experimental evidence suggesting that high dose co-trimoxazole might interfere with normal somatic growth, ${ }^{2}$ we studied the growth velocity and longitudinal growth of girls receiving prophylactic co-trimoxazole over long periods.

\section{Methods and results}

A total of 114 girls aged 2-12 years were given continuous low dose cotrimoxazole (about $10 \mathrm{mg}$ sulphamethoxazole and $2 \mathrm{mg}$ trimethoprim $/ \mathrm{kg} / \mathrm{day}$ ) for between six months and six years after a urinary infection. Their heights and weights were measured accurately and the urine was cultured every three months. The intravenous urogram and micturating cystourethrogram were normal in 54 girls; abnormalities included vesicoureteric reflux in 51 girls, with renal scarring in 12 and miscellaneous lesions in nine (scars four, duplex systems two). Girls with neuropathic bladder or outflow obstruction were excluded. Height and weight velocities were calculated over six month periods randomly distributed over the calendar year. Velocities were grouped in one year age groups according to the age at the start of the period of prophylaxis.

Growth velocities obtained in 114 girls both while they were having and were not having co-trimoxazole prophylaxis were first compared with standard growth velocity curves. ${ }^{3}$ No deviation from the normal in weight or height was found in either of the samples (figure). Two way non-orthogonal

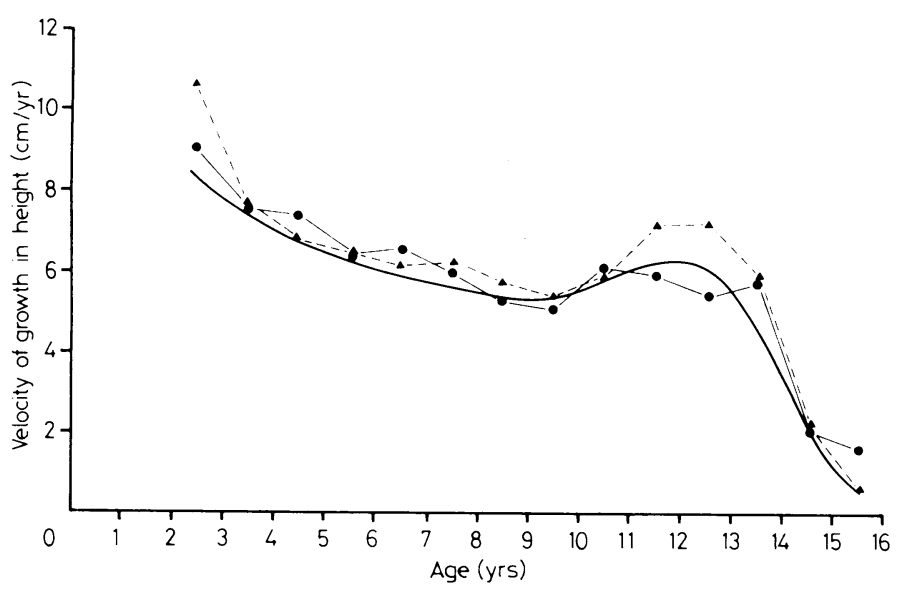

Height velocity curves in 114 girls, during $(\boldsymbol{\Delta})$ and before and after ( co-trimoxazole prophylaxis calculated over six month periods compared with standard height velocity. ${ }^{3}$

analysis of variance with age group and treatment group as main effects was then used to assess the effect of treatment. ${ }^{4}$ There was a significant effect of age on both height and weight, but when allowance was made for this there was no overall difference between treatment groups (with or without prophylaxis). There was, however, a barely significant effect $(p<0.05)$ on growth velocity in early adolescence, when growth was slightly faster in girls receiving co-trimoxazole.

Data from a further subset of 53 girls, where a six month growth velocity was calculable both before and immediately after a change in treatment, were analysed with a crossover design. ${ }^{5}$ Some 29 girls changed from no treatment to co-trimoxazole prophylaxis and 24 changed the reverse way. No significant interaction was found between weight or height velocity and whether treatment was given or not apart from the inevitable one of slight difference of age between the two groups, indicating that the order in which treatment was given was unimportant as regards growth. Thus growth velocities over six month periods were satisfactory during co-trimoxazole prophylaxis even in 51 girls with vesicoureteric reflux and 16 with renal scarring.

The effect of duration of co-trimoxazole prophylaxis on growth was further studied in 46 girls with vesicoureteric reflux with or without renal scarring who received continuous prophylaxis for two to five years. They were divided into three cohorts according to the duration of prophylaxis: 46 girls had at least two years of treatment, 35 at least three years, and 22 at least four years. Because of the wide age variation heights were expressed as standard deviation scores. No significant change in height status from the start to the end of co-trimoxazole prophylaxis occurred during or between any time periods.

Six children developed a single asymptomatic reinfection without any effect on growth.

\section{Comment}

From our results we may conclude that during low dose cotrimoxazole prophylaxis of urinary infection growth velocity remains normal. Normal somatic growth may also be expected during long periods of treatment with low dose co-trimoxazole in girls without urinary outflow obstruction, including those with vesicoureteric reflux.

We thank Sister Jo March and her staff for measuring the children, Dr Gary Katz for help in the clinic, and Mrs Trudy Clark for secretarial help. The work was supported by grants from the Medical Research Council and the National Kidney Research Fund.

1 Smellie JM, Katz G, Grüneberg RN. Controlled trial of prophylactic treatment in childhood urinary tract infection. Lancet 1978;ii:175-8.

${ }^{2}$ Udall V. Toxicology of sulphonamide-trimethoprim combinations. Postgrad Med F 1969;45, suppl:42-5

3 Tanner JM, Whitehouse RH, Takaishi $M$. Standards from birth to maturity for height, weight, height velocity, and weight velocity: British children, 1965 -I and -II. Arch Dis Child 1966;41:454-71, 613-35.

4 Finn JD. Multivariances: univariate and multivariate analysis of variances, covariance and regression. Ann Arbor, Michigan: National Education Resources, 1972.

${ }^{5}$ Hills $\mathrm{M}$, Armitage $\mathrm{P}$. The two-period cross-over clinical trial. $\mathrm{Br} \mathcal{F} \mathrm{Clin}$ Pharmacol 1979;8:7-20.

(Accepted 27 May 1983)

University College Hospital, London WC1E 6 AO

JEAN M SMELLIE, DM, FRCP, senior lecturer in paediatrics and honorary consultant paediatrician

Institute of Child Health and The Hospital for Sick Children, London WC1 N1EH

MICHAEL A PREECE, MD, FRCP, reader in growth and development, and consultant paediatrician

The Wellcome Research Laboratories, Beckenham, Kent

A M PATON, BSC, lately research assistant

Correspondence to: Dr Jean $M$ Smellie.

\section{Stump pemphigoid: a new complication of the amputee}

Skin problems affecting the stump of the leg amputee are common, and diabetic patients have additional factors which may cause complications during rehabilitation. We report a case of a diabetic amputee who developed a previously unreported skin complication-localised bullous pemphigoid.

\section{Case report}

A 66 year old man presented to his general practitioner with a three month history of an infected ulcer on the great toe of the right foot. This developed without blistering and was consistent with the ulceration classically seen in the diabetic foot. He was noted to have glycosuria on routine urine examination, and the blood glucose concentration was $22 \mathrm{mmol} / \mathrm{l}(400 \mathrm{mg} / 100 \mathrm{ml})$. He was started on a carbohydrate restricted diet and referred to hospital as the ulcer failed to heal.

Examination showed a deep ulcer on the great toe with spread to the underlying bone. There were no pulses palpable below the right femoral artery but he had no evidence of peripheral neuropathy. Ultrasound investi- 